\title{
SINTESIS DE POLIURETANOS A PARTIR DE POLIOLES OBTENIDOS A PARTIR DEL ACEITE DE HIGUERILLA MODIFICADO POR TRANSESTERIFICACIÓN CON PENTAERITRITOL
}

\author{
Manuel F. Valero*, Jorge E. Pulido y Álvaro Ramírez \\ Grupo de Investigación en Polímeros, Escuela de Ingeniería Química, Universidad Industrial de Santander A.A. 678, Bucaramanga, \\ Colombia \\ Zhengdong Cheng
}

Chemical Engineering Department, Texas A\&M University, College Station, 77840, TX, USA

Recebido em 24/4/08; aceito em 20/6/08; publicado na web em 10/11/08

\begin{abstract}
POLYURETHANES SINTETIZED OF POLYOLS OBTAINED FROM CASTOR OIL MODIFIED BY TRANSESTERIFICATION WITH PENTAERYTHRITOL. Castor oil was reacted by transesterification with various percentages in mass of pentaerythritol to obtain different esters of pentaerythritol. Alternatively, glycerol was also used instead of pentaerythritol for the same reaction in order to establish comparative reference products. The products of the reactions were characterized by matrix-assisted laser desorption/ ionization time-of-flight mass spectroscopy in order to detect and quantify (in terms of the molecular mass and structural information) the components of the products obtained. Analysis for hydroxyl value, acid value, viscosity and specific gravity were used to complete the characterization of the polyols obtained and also of the original castor oil. The polymer characterization was accomplished by tensile stress-strain tests, Shore A hardness, thermogravimetric analysis and chemical resistance to solvents.
\end{abstract}

Keywords: castor oil transesterification; MALDI TOF mass spectroscopy; polyurethanes.

\section{INTRODUCCIÓN}

El aceite de higuerilla se extrae de la semilla de la planta " $R i$ cinus communis" y se caracteriza por ser uno de los pocos aceites vegetales cuya composición es casi la de un solo componente, puesto que el $90 \%$ corresponde al triglicérido del ácido ricinoléico. Desde el punto de vista de la innovación, la estructura química del aceite de higuerilla es de gran interés, porque permite un gran número de reacciones y productos derivados. ${ }^{1}$

El uso del aceite de higuerilla en la síntesis de elastómeros de poliuretano e IPNs ha sido reportado en numerosas investigaciones..$^{2-4}$ Estos estudios muestran que se puede obtener un amplio rango de propiedades físicas y mecánicas dependiendo del contenido del poliol y el diisocianato. La composición y la relación NCO/OH de los segmentos del poliuretano afectan la estructura, organización y flexibilidad del polímero, y por consiguiente la morfología y las propiedades mecánicas. Sin embargo, los materiales obtenidos a partir del aceite de higuerilla presentan propiedades como bajo módulo de Young, baja resistencia al rasgado y también baja resistencia a tratamientos a alta temperatura..$^{5-7}$

Una forma de modificar estas propiedades es la transesterificación del aceite con alcoholes polihidroxílicos para introducir grupos hidroxílicos reactivos. Al aumentar estos grupos se incrementa el grado de entrecruzamiento del poliuretano, por lo que las propiedades del poliuretano cambian. El término transesterificación se usa generalmente para describir una clase

de reacciones orgánicas donde un éster es transformado en otro a través del intercambio del grupo alcoxi. ${ }^{8}$ En la transesterificación de aceites vegetales, un triglicérido reacciona con un alcohol, en presencia de un ácido o base fuerte, produciendo una mezcla de ésteres alquílicos de ácidos grasos y glicerol. El proceso total es una secuencia de 3 reacciones consecutivas irreversibles en la cual los di- y monoglicéridos se forman como productos intermedios.

El estudio detallado de la síntesis de poliuretanos e IPNs a partir

*e-mail: mfermando4@yahoo.com del aceite de higuerilla modificado no ha recibido mucha atención. En la literatura se encuentran publicados trabajos que muestran el uso de glicerol para la transesterificación del aceite de higuerilla. ${ }^{7-9}$ Los resultados muestran un incremento en las propiedades tensiles, dureza y resistencia al ataque químico debido un mayor grado de entrecruzamiento ocasionado por el incremento en el contenido de grupos hidroxilo. Sin embargo, cuando se usa glicerol para la transesterificación, el triglicérido obtenido posee baja estabilidad térmica y es fácilmente descompuesto a altas temperaturas. Esta desventaja se debe a la presencia de átomos de hidrógeno en la posición $\beta$ relativos a los grupos hidroxilo del glicerol. ${ }^{9}$ Este problema puede solucionarse utilizando otro polialcohol que no contenga átomos de hidrógeno en la posición $\beta$, por ejemplo pentaeritritol.

En el presente trabajo se buscó aprovechar el potencial del aceite de higuerilla mediante reacciones de transesterificación con pentaeritritol para generar un nuevo poliol con funcionalidad y estructura química diferente. Se determinaron las propiedades fisicoquímicas de los polioles obtenidos en función de la cantidad de agente modificador utilizado. El trabajo abordó el estudio de los productos de la reacción de transesterificación del aceite mediante espectroscopia de masas MALDI TOF. Esta técnica es de reciente aplicación para caracterizar aceites. ${ }^{10}$ Se destaca que su utilización en esta investigación resultó muy adecuada para establecer la composición de los polioles desarrollados. Los polioles obtenidos a partir de la reacción de transesterificación del aceite de higuerilla con pentaeritritol fueron utilizados en la síntesis de poliuretanos. Los poliuretanos obtenidos se caracterizaron mediante análisis termogravimétrico (TGA), tensióndeformación, dureza Shore A y resistencia al ataque químico.

\section{PARTE EXPERIMENTAL}

\section{Materiales}

Aceite de higuerilla (índice de hidroxilo: $163 \mathrm{mg} \mathrm{KOH} / \mathrm{g}$ e índice de acidez: 2,33 mg KOH/g) grado USP, distribuido por laboratorios León, Bucaramanga. Pentaeritritol marca Fisher, distribuido por 
Produquímica de Colombia S.A. Los reactivos utilizados para la caracterización fueron de grado analítico marca Aldrich, distribuidos por Arquilab Ltda. Metilen difenil diisocianato (MDI) utilizado en la síntesis de los poliuretanos, marca Aldrich, distribuido por Arquilab Ltda, Bucaramanga.

\section{Métodos}

\section{Modificación del aceite de higuerilla por transesterificación con pentaeritritol}

La transesterificación del aceite de higuerilla usando pentaeritritol se realizó en un balón reactor de cuatro bocas equipado con termómetro, agitador mecánico, atmósfera inerte y condensador de reflujo. El balón reactor se cargó con el aceite de higuerilla, pentaeritritol y óxido de plomo $(\mathrm{PbO})$, en relación $0.05 \%(\mathrm{PbO} /$ masa de aceite). El óxido de plomo actúa como catalizador y rompe los enlaces que unen a los ácidos grasos del triglicérido. 6 Para encontrar las condiciones de reacción se partió de las condiciones reportadas por Prashantha y colaboradores para aceite de higuerilla transesterificado con glicerol, además de tener en cuenta restricciones como la temperatura de sublimación del pentaeritritol $\left(220^{\circ} \mathrm{C}\right){ }^{7}$ Se hicieron varios experimentos de síntesis variando la temperatura y tiempo de reacción, y se midió el índice de hidroxilo del poliol resultante. A partir de estos resultados experimentales iniciales se fijaron como condiciones de reacción $210{ }^{\circ} \mathrm{C}+/-5^{\circ} \mathrm{C}$, por $2 \mathrm{~h}$. Se realizó la transesterificación del aceite con pentaeritritol utilizando las composiciones que se describen en la Tabla 3. También, se efectuó la transesterificación del aceite con glicerol (bajo las mismas condiciones), con el fin de comparar los resultados obtenidos en trabajos previos. ${ }^{10-13}$

Síntesis de los elastómeros de poliuretano a partir de los polioles derivados del aceite de higuerilla modificado con pentaeritritol

El poliol se mezcló con el metilen difenil diisocianato (MDI) fundido. El producto se agitó por 5 min para obtener una mezcla homogénea. El prepolímero obtenido se sometió a vacío y se vertió en un molde de acero hermético de placas paralelas. El período de curado constó de dos etapas, la primera a temperatura ambiente y la segunda a $90^{\circ} \mathrm{C}$, ambas durante $4 \mathrm{~h}$. Se utilizó para la síntesis de los poliuretanos una relación $\mathrm{NCO} / \mathrm{OH}=0.7$.

\section{Caracterización de los polioles}

\section{Espectroscopia de masas MALDI TOF}

En este trabajo se utilizó espectroscopia de masas tipo MALDI TOF MS Matrix Assisted Laser Desorption/Ionization Time of Flight Mass Spectroscopy con el fin de detectar y caracterizar (en términos de la masa molecular y información estructural) los productos obtenidos a partir de las rutas de modificación del aceite planteadas. La técnica registra los cambios en la velocidad con que viajan las partículas - por un tubo al vacío - y los relaciona con la masa molecular y la carga.

Se utilizó un espectroscopio de masas ABI 4700 TOF-TOF con láser de nitrógeno. Se usó un voltaje de aceleración de de 25 $\mathrm{kV}$. Los iones positivos fueron detectados con modo reflectron $(20$ $\mathrm{kV}$ ). El láser de nitrógeno fue operado a $4 \mathrm{~Hz}$. A una solución de $\mathrm{NaCl}$ (el $\mathrm{NaCl}$ se adicionó para mejorar la ionización de la mezcla matriz/muestra) se adicionaron la muestra y la solución de la matriz orgánica (se utilizó 2,5-ácido di-hidroxibenzoico (DHB)); la matriz y la muestra se mezclaron en una proporción de 5:2 matriz/muestra. Se depositaron $0.5-1 \mu \mathrm{L}$ de muestra en solución $(\mathrm{NaCl}+$ mezcla muestra y matriz). El rango de masas utilizado fue entre 100-1500 $\mathrm{m} / \mathrm{z}$ (masa/carga). ${ }^{9}$
Índice de hidroxilo y valor ácido

Se determinó el índice de hidroxilo de los polioles obtenidos según la norma ASTM D1957-86, con el fin de medir el aumento en el número de grupos $\mathrm{OH}$ de los polioles obtenidos a partir de la modificación el aceite de higuerilla.

El índice de hidroxilo se calculó según la siguiente ecuación:

Valor hidroxilo $=\frac{B+\left(\left(S^{*} A\right) / C\right)-V}{S} N * 56.1 \quad[\mathrm{mg} \mathrm{KOH} / \mathrm{g}$ muestra $]$

donde: $A$ = Solución de $\mathrm{KOH}$ requerida para la titulación del valor ácido $(\mathrm{mL}) ; B=$ Solución de $\mathrm{KOH}$ requerida para la titulación del blanco $(\mathrm{mL}) ; C=$ Muestra usada para el valor ácido $(\mathrm{g}) ; V=$ Solución de $\mathrm{KOH}$ requerida para la titulación de la muestra acetilada $(\mathrm{mL})$; $S=$ Muestra usada para la acetilación (g).

Se determinó el índice de acidez según la norma ASTM D466287, con el fin de medir los constituyentes ácidos del poliol obtenidos en la transesterificación. La acidez (contenido de ácidos grasos libres) del aceite es una medida del grado en que la hidrólisis ha liberado a los ácidos grasos de su enlace éster con una molécula de glicérido original. En parte es por esto que la acidez se conoce comúnmente como el porcentaje de contenido de ácidos grasos libres (FFA en ingles). Cuando hay mezclas de aceites involucrados - como en el caso actual - se prefiere usar el valor ácido (VA) que se define como el número de $\mathrm{mg}$ de hidróxido de potasio requeridos para neutralizar los ácidos libres en un gramo de muestra.

Viscosidad y gravedad específica

La viscosidad fue medida en un viscosímetro Brookfield, modelo DV - III + Rheometer, a $200 \mathrm{rpm}$, utilizando aguja tamaño 31 y la gravedad específica se determinó según la norma ASTM D792-86.

\section{Caracterización de los poliuretanos}

\section{Resistencia al ataque químico}

Se evaluó según norma ASTM D543-67. Las muestras tomadas fueron cilindros de $1.6 \mathrm{~cm}$ de diámetro y $0.3 \mathrm{~cm}$ de espesor. Los reactivos usados para tal fin fueron: acetona, agua destilada (a temperatura ambiente y a $70{ }^{\circ} \mathrm{C}$ ), tolueno, amoniaco comercial, ácido clorhídrico al 10\%, gasolina y tetracloruro de carbono. Las muestras se sumergieron en cada solvente por 7 días. Durante este tiempo se registraron cambios en la superficie de las muestras. Al final de los 7 días las muestras se secaron y se removió el exceso de solvente. Las muestras permanecieron durante $24 \mathrm{~h}$ en un desecador. Este procedimiento se llevó a cabo en al menos 3 muestras de cada material. El porcentaje de absorción fue calculado usando la siguiente ecuación: ${ }^{12}$

$\%$ Hinchamiento $=\frac{m t-m i}{m i} * 100$

donde: $\mathrm{mt}=$ masa de la muestra en el tiempo $\mathrm{t}$ (7 días en este caso) $\mathrm{y} \mathrm{mi}=$ masa de la muestra inicial

\section{Análisis termogravimétrico (TGA)}

El comportamiento térmico de las muestras se investigó por termogravimetría (la masa de una sustancia es medida como una función de la temperatura mientras la sustancia es sujeta a un programa de temperatura controlada). El análisis termogravimétrico se llevó a cabo en una termobalanza TA 2050 TGA. La velocidad de calentamiento fue de $10^{\circ} \mathrm{C} / \mathrm{min}$ en un intervalo de temperatura entre 25-700 ${ }^{\circ} \mathrm{C}$. Los ensayos se realizaron bajo atmósfera de nitrógeno 
Tensión-deformación y dureza shore A

Las pruebas de resistencia a tensión se realizaron en una máquina de tensión universal INSTRON. Se utilizaron mordazas cilíndricas que sostenían la muestra. La velocidad de prueba fue $0.2 \mathrm{in} / \mathrm{min}$ a una temperatura $19+/-2{ }^{\circ} \mathrm{C}$, según la norma ASTM D638. Las dimensiones de la muestra fueron láminas de $40 \mathrm{~mm} * 6 \mathrm{~mm} * 3$ $\mathrm{mm}$. Para cada muestra de 3 a 5 replicas fueron evaluadas en las mismas condiciones. La dureza de los materiales se determinó según la norma ASTM D785. Se determinó utilizando un durómetro shore A con punzón, y se tomaron medidas en al menos 10 puntos dentro de la lámina del material.

\section{RESULTADOS Y DISCUSION}

\section{Caracterización de polioles}

\section{Espectroscopia de masas MALDI TOF}

En la Figura 1 se observa el espectro de masas MALDI TOF del aceite de higuerilla sin modificación. La Tabla 1 muestra la masa observada y la masa calculada de todos los componentes identificados por espectroscopia de masas MALDI TOF del aceite de higuerilla. El principal pico que aparece a $955.65 \mathrm{~m} / \mathrm{z}$ corresponde a la tri-ricinoleína, el más abundante triglicérido del aceite de higuerilla. Los picos de mediana intensidad entre un $\mathrm{m} / \mathrm{z}$ de 675 y 600 corresponden al diglicérido del ácido ricinoleico. Se observa un pico de mediana intensidad a $374 \mathrm{~m} / \mathrm{z}$ que corresponde al monoglicérido del ácido ricinoleico.

También aparecen dos picos de mediana intensidad a 271 y $220 \mathrm{~m} / \mathrm{z}$ que corresponden al ácido esteárico y al ácido palmítico, respectivamente, menos dos moléculas de agua (pérdida del grupo OOH). Los picos entre 100 y $200 \mathrm{~m} / \mathrm{z}$ posiblemente son compuestos minoritarios del aceite de higuerilla. Esta conjetura fue validada, ya que estos picos permanecen inmodificables después de la transesterificación (obsérvese en el segundo espectro del poliol 1, como estos picos se repiten, exactamente al mismo $\mathrm{m} / \mathrm{z}$ ). Finalmente se observan algunos picos de baja intensidad que corresponden a triglicéridos de ésteres de ácidos grasos en los cuales una cadena de ácido ricinoleico es reemplazada por una cadena de algún componente minoritario del aceite de higuerilla, como el ácido linoleico o el oleico., ${ }^{9,10}$

Las fracciones en masa de los componentes del aceite de higuerilla son las siguientes: $77 \%$ tri-ricinoleína, 14\% di-ricinoleína, tri-ricinoleína + di-ricinoleína y $9 \%$ de componentes minoritarios del aceite de higuerilla. Los resultados indican que cerca del $91 \%$ del aceite corresponde a los glicéridos del ácido ricinoleico.

En la Figura 2 se encuentra el espectro para el poliol 1. Se ob-

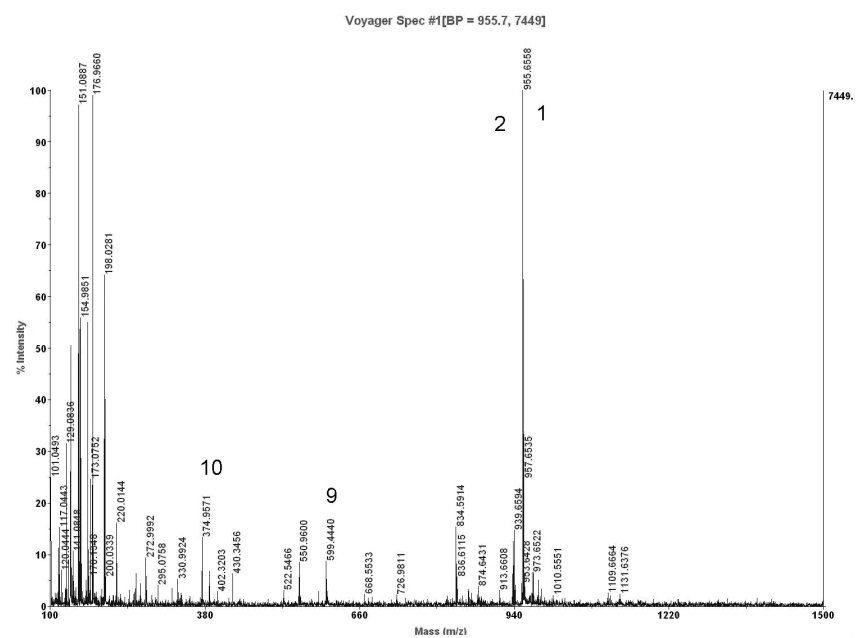

Figura 1. Espectro de masas del aceite de higuerilla sin modificar

serva un pico de muy baja intensidad a $955 \mathrm{~m} / \mathrm{z}$, correspondiente al triglicérido del ácido ricinoleico; en contraste, crecen en intensidad los picos correspondientes al diglicérido y al monoglicérido para valores m/z de 675 y 395 respectivamente. Aparecen dos picos de mediana intensidad a valores $\mathrm{m} / \mathrm{z}$ de 719 y 411 , relacionados con el di-ricinoleato de pentaeritritol y el mono-ricinoleato de pentaeritritol. Se observan picos de baja intensidad a 439, 403 y 344 que corresponden al mono-ricinoleato de pentaeritritol $+\mathrm{Na}$, monoricinoleato de pentaeritritol $+\mathrm{Na}-2 \mathrm{H}_{2} \mathrm{O}$ y mono-ricinoleato de pentaeritritol $-4 \mathrm{H}_{2} \mathrm{O}$. Los componentes identificados del espectro se muestran en la Tabla 2. La aparición de estos picos evidencia la transesterificación del aceite con el pentaeritritol.

Las fracciones en masa de los productos de la reacción de transesterificación del aceite de higuerilla con pentaeritritol fue la siguiente: aproximadamente $0 \%$ tri-ricinoleína, $20.8 \%$ di-ricinoleína, $40.5 \%$ mono-ricinoleína, $8.5 \%$ di-ricinoleato de pentaeritritol, $21.2 \%$ mono-ricinoleato de pentaeritritol y $9 \%$ componentes minoritarios (permanecen invariantes después de la reacción transesterificación, debido a la ausencia de grupos $\mathrm{OH}$ en estos componentes).

\section{Esquema de la reacción de transesterificación del aceite de} higuerilla con pentaeritritol

En el esquema de la Figura 3 se muestran los productos de la reacción de transesterificación del aceite de higuerilla con pentaeritritol. Se encontró que, entre los ricinoleatos de pentaeritritol, el

Tabla 1. Componentes individuales identificados por espectroscopia de masas MALDI TOF del aceite de higuerilla

\begin{tabular}{lcccc}
\hline Número & Estructura & $\mathrm{m} / \mathrm{z}$ Observada & $\mathrm{m} / \mathrm{z}$ Calculada & Intensidad \\
\hline 1 & tri-ricinoleina $+\mathrm{Na}+\mathrm{H}$ & 957.649 & 956 & 0.79 \\
2 & tri-ricinoleina $+\mathrm{Na}$ & 955.6479 & 955 & 0.95 \\
3 & tri-ricinoleina $+\mathrm{Na}+\mathrm{H}-\mathrm{H} 2 \mathrm{O}$ & 939.6531 & 938 & 0.165 \\
4 & tri-ricinoleina $+\mathrm{Na}+\mathrm{H} 2 \mathrm{O}$ & 937.6385 & 937 & 0.28 \\
5 & tri-ricinoleina $-\mathrm{H} 2 \mathrm{O}$ & 913.6388 & 914 & 0.08 \\
6 & tri-ricinoleina $+\mathrm{H}-3 \mathrm{H} 2 \mathrm{O}$ & 879.6540 & 879 & 0.03 \\
7 & di-ricinoleina $+\mathrm{Na}$ & 675.4475 & 675 & 0.05 \\
8 & di-ricinoleina $+\mathrm{H}-2 \mathrm{H} 2 \mathrm{O}$ & 617.4396 & 617 & 0.05 \\
9 & di-ricinoleina $+\mathrm{H}-3 \mathrm{H} 2 \mathrm{O}$ & 599.4442 & 399 & 0.17 \\
10 & mono-ricinoleina $+\mathrm{H}$ & 374.9571 & 273 & 0.14 \\
11 & Ácido esteárico $+\mathrm{Na}-2 * \mathrm{H} 2 \mathrm{O}$ & 272.9992 & 220.4 & 0.085 \\
\hline
\end{tabular}




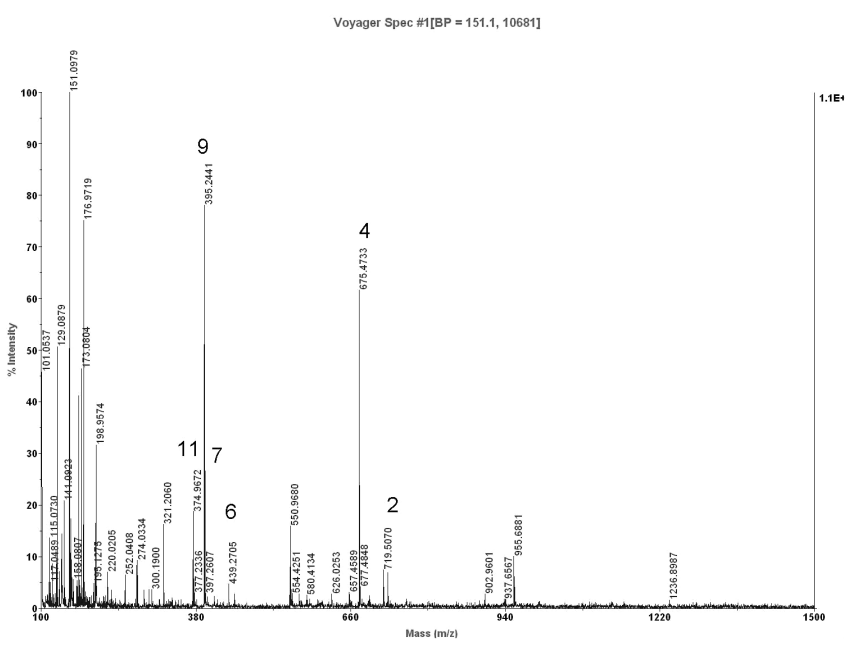

Figura 2. Espectro de masas del poliol 1, por MALDI TOF en el rango de $100-1500 \mathrm{~m} / \mathrm{z}$

más abundante es el mono-ricinoleato y que entre los glicéridos del ácido ricinoleico el más abundante, es el monoglicérido del ácido ricinoleico.

\section{Índice de hidroxilo y valor ácido}

En la Figura 4 se muestran los valores del índice de hidroxilo para los polioles obtenidos con diferente adición del tipo de modificador (pentaeritritol o glicerina) usado en la transesterificación del aceite de higuerilla, con respecto al porcentaje de agente modificador. Los resultados del índice de hidroxilo de los polioles obtenidos a través de la modificación del aceite con glicerol coinciden con los obtenidos en trabajos previos. ${ }^{11}$ Se alcanzaron valores más altos del índice de hidroxilo cuando el aceite se modificó con pentaeritritol. Este resultado se debe a que los productos de la reacción de transesterificación del aceite de higuerilla con glicerol contienen 3 grupos $\mathrm{OH} ;{ }^{13}$ mientras que, los productos de la reacción de transesterificación del aceite con pentaeritritol poseen una funcionalidad de 4 o incluso mayor (ver ésteres de pentaeritritol, Figura 3).

En la Figura 4 también se observa que el índice de hidroxilo de los polioles Penta aumenta a medida que se incrementa el porcentaje de pentaeritritol; sin embargo, los polioles que contenían un porcentaje superior al $6 \%$ presentaban un precipitado. Este preci- pitado se analizó utilizando espectroscopia de infrarrojos FTIR y se encontró que era pentaeritritol sin reaccionar, por lo que solamente se sintetizaron polioles hasta un porcentaje máximo de pentaeritritol del 6\%. Para los polioles obtenidos a partir del aceite modificado con glicerol - polioles Glice - se observa que índice de hidroxilo aumenta en función de la cantidad de agente modificador hasta un porcentaje del $6 \%$. Para porcentajes superiores al $6 \%$ el índice de hidroxilo de los polioles no varía significativamente.

La acidez del aceite es una medida del grado en que la hidrólisis ha liberado a los ácidos grasos de su enlace éster con una molécula de glicérido original. Cuando hay mezclas de aceites involucrados - como en el caso nuestro - se prefiere usar el valor ácido (VA) que se define como el número de mg de hidróxido de potasio requeridos para neutralizar los ácidos libres en $1 \mathrm{~g}$ de muestra. Se encontró que no existe una diferencia significativa - según la norma ASTM D4662-87 - en el valor ácido de los polioles obtenidos del aceite modificado con pentaeritritol y glicerol. Este resultado puede deberse a que la cantidad de ácidos libres del aceite de higuerilla no cambia durante la transesterificación (el resto de ácidos grasos del aceite de higuerilla como el ácido linoleico, el ácido palmítico y el ácido oleico no poseen grupos $-\mathrm{OH}$ en su estructura). Los valores se reportan en la Tabla 3 .

Los resultados obtenidos en este estudio, cuando el aceite de higuerilla se modificó con glicerol, son consistentes con los resultados disponibles en la literatura.

\section{Viscosidad y gravedad específica}

Los valores de viscosidad y gravedad específica de los polioles obtenidos por transesterificación, en función de la cantidad de agente modificador empleado, se reportan en la Tabla 3. La viscosidad de los polioles obtenidos mediante la modificación del aceite con pentaeritritol se incrementa a medida que aumenta la cantidad de pentaeritritol. Mientras que la viscosidad de los polioles obtenidos por la modificación del aceite con glicerol aumenta inicialmente, pero luego permanece aproximadamente constante; el incremento en la viscosidad puede ser atribuido a la presencia de grupos polares que aumentan las interacciones entre las moléculas. La gravedad específica de los polioles obtenidos aumentó en comparación con la del aceite de higuerilla sin modificar. La gravedad específica de los polioles Penta es mayor que para los polioles Glice; este comportamiento obedece a que la densidad del pentaeritritol es mayor que la densidad del glicerol. Los valores reportados de gravedad específica de los polioles se encuentran en el intervalo entre 0.955 y 0.980 .

Tabla 2. Componentes individuales identificados por espectroscopia de masas MALDI TOF del poliol 1

\begin{tabular}{lcccc}
\hline Número & Estructura & $\mathrm{m} / \mathrm{z}$ Observada & $\mathrm{m} / \mathrm{z}$ Calculada & Intensidad \\
\hline 1 & tri-ricinoleina $+\mathrm{Na}$ & 956.6736 & 955 & 0.03 \\
2 & di-ricinoleato de pentaeritritol $+\mathrm{Na}$ & 719.4822 & 719 & 0.1 \\
3 & di-ricinoleato de pentaeritritol $+\mathrm{Na}+\mathrm{H}$ & 720.5450 & 720 & 675 \\
4 & di-ricinoleina $+\mathrm{Na}$ & 675.4583 & 657 & 0.05 \\
5 & di-ricinoleina $+\mathrm{H}-\mathrm{H} 2 \mathrm{O}$ & 657.4527 & 439 & 0.05 \\
6 & mono-ricinoleato de pentaeritritol $+\mathrm{Na}$ & 439.2469 & 416 & 0.06 \\
7 & mono-ricinoleato de pentaeritritol & 411.2016 & 403 & 0.12 \\
8 & mono-ricinoleato de pentaertritol $+\mathrm{Na}-2 \mathrm{H} 2 \mathrm{O}$ & 405.0971 & 395 & 0.05 \\
9 & mono-ricinoleina $+\mathrm{Na}$ & 395.2292 & 373 & 0.99 \\
10 & mono-ricinoleina $+\mathrm{H}$ & 374.9695 & 344 & 0.08 \\
11 & mono-ricinoleato de pentaeritritol $+\mathrm{Na}-4 \mathrm{H} 2 \mathrm{O}$ & 342.6783 & 271 & 0.05 \\
13 & Ácido esteárico $+\mathrm{Na}-2 * \mathrm{H} 2 \mathrm{O}$ & 272.9992 & 220.4 & 0.085 \\
\hline
\end{tabular}




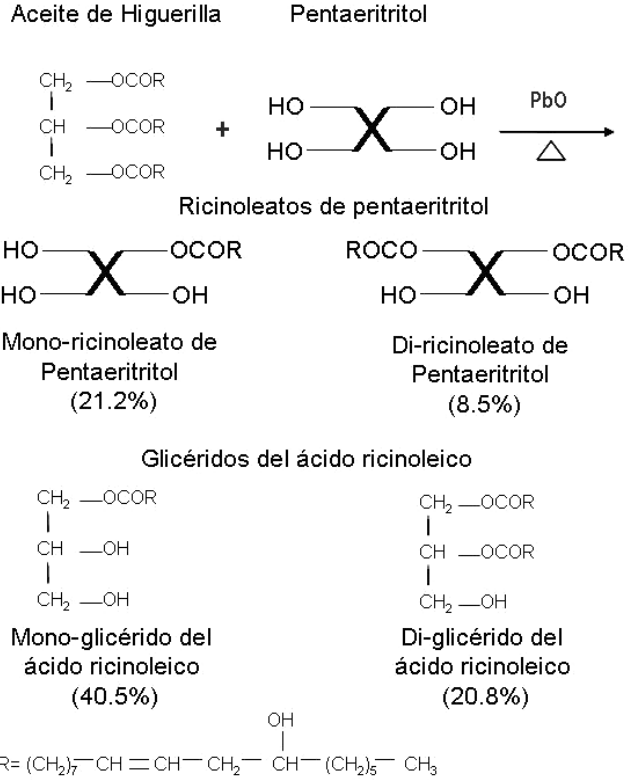

Figura 3. Productos de la reacción de transesterificación del aceite de higuerilla usando pentaeritritol (el valor entre paréntesis indica la fracción en masa de cada componente estimada por espectroscopia de masas MALDI TOF)

\section{Caracterización de poliuretanos}

En la Figura 5 y Figura 6 se observan los esquemas de la reacción de obtención del poliuretano a partir del aceite de higuerilla sin modificar y a partir del poliol obtenido por transesterificación del aceite de higuerilla con pentaeritritol, respectivamente. En los esquemas se evidencia que al cambiar la estructura química del poliol se modifica la estructura poliuretano. También, se observa en la Figura 6 la forma como se establecen los enlaces tipo puentes de hidrógeno entre los segmentos del poliuretano (interacciones intra- e inter-moleculares dentro de la matriz del polímero entre los grupos carbonilo $\mathrm{C}=\mathrm{O}$ del grupo éster y los hidrógenos del grupo $-\mathrm{NH}-$ ), aspecto amplificado que se muestra en la Figura 7.

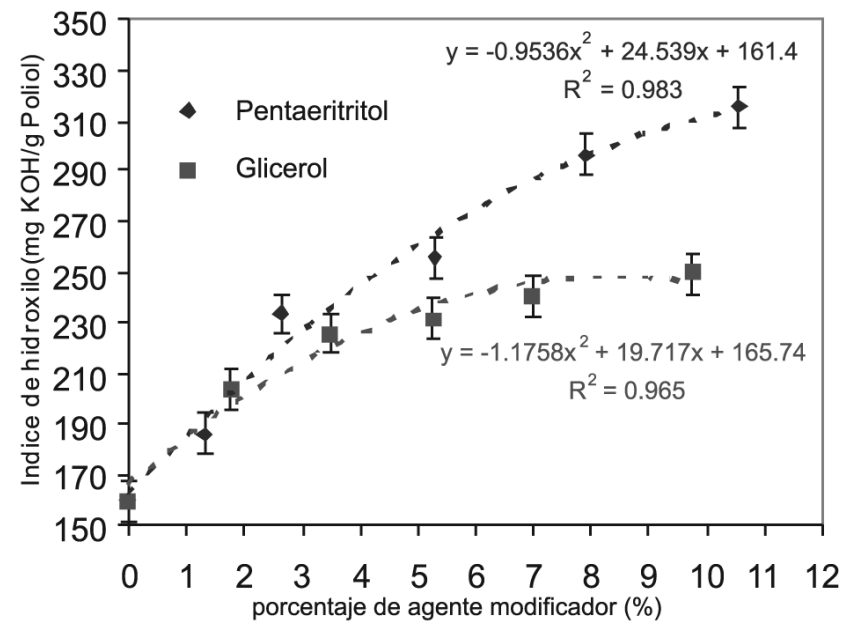

Figura 4. Índice de hidroxilo del aceite de higuerilla y los polioles obtenidos por transesterificación con pentaeritritol (polioles Penta) y glicerina (polioles Glice)

\section{Resistencia al ataque químico}

Los resultados del ensayo de resistencia química de los poliuretanos sintetizados frente a diferentes solventes se determinó que todos los poliuretanos obtenidos muestran resistencia a ácidos y bases (hinchamiento bajo en: ácido clorhídrico al 10\%, ácido acético e hidróxido de sodio al 10\%); se determinó que los poliuretanos no se ven afectados cuando son inmersos en agua bajo el tiempo específico de la prueba. También se pudo determinar que los poliuretanos presentaban el mayor grado de hinchamiento en acetona, atribuible a la polaridad de este solvente.

Se observa en la Figura 8 el porcentaje de hinchamiento de los poliuretanos sintetizados a partir del aceite modificado con pentaeritritol. Todos los poliuretanos presentaron el mayor grado de hinchamiento en acetona atribuible a la polaridad de este solvente. El máximo hinchamiento lo presentó el poliuretano obtenido del Penta 0 (PU-P0-0.7), mientras que el mínimo lo registró el poliuretano obtenido del Penta 2 (PU-P2-0.7). Al incrementar la funcionalidad

Tabla 3. Índice de hidroxilo, índice de acidez, viscosidad y gravedad específica de los productos obtenidos en función de la concentración de agente modificado (pentaeritriol o glicerina)

\begin{tabular}{lccccc}
\hline Nombre del poliol & $\begin{array}{c}\text { Porcentaje en masa de } \\
\text { agente modificador }(\%)\end{array}$ & $\begin{array}{c}\text { índice de hidroxilo } \\
(m g \text { KOH/g muestra })\end{array}$ & $\begin{array}{c}\text { Índice de acidez } \\
(\mathrm{mgKOH} / \text { gmuestra })\end{array}$ & Viscosidad $^{a}(\mathrm{cp})$ & Densidad $^{b}\left(\mathrm{~g} \mathrm{~mL}^{-1}\right)$ \\
\hline Pentaeritriol & & & & & \\
\hline Penta 0 (P0) & 1.32 & $191 \pm 3$ & $1.05 \pm 0.03$ & $572 \pm 5$ & $0.958 \pm 0.004$ \\
Penta 1 (P1) & 2.64 & $236 \pm 3$ & $0.71 \pm 0.03$ & $732 \pm 5$ & $0.966 \pm 0.004$ \\
Penta 2 (P2) & 5.28 & $250 \pm 3$ & $0.80 \pm 0.03$ & $835 \pm 5$ & $0.971 \pm 0.004$ \\
Penta 3 (P3) & 7.92 & $297 \pm 3$ & $1.22 \pm 0.03$ & $924 \pm 5$ & $0.977 \pm 0.004$ \\
Penta 4 (P4) & 10.56 & $315 \pm 3$ & $1.08 \pm 3$ & $992 \pm 5$ & $0.980 \pm 0.004$ \\
\hline Glicerina & & & & $660 \pm 5$ & $0.965 \pm 0.004$ \\
Glice 1 (G1) & 1.75 & $201 \pm 3$ & $0.52 \pm 0.03$ & $668 \pm 5$ & $0.965 \pm 0.004$ \\
Glice 2 (G2) & 3.5 & $219 \pm 3$ & $0.47 \pm 0.03$ & $649 \pm 5$ & $0.968 \pm 0.004$ \\
Glice 3 (G3) & 5.25 & $222 \pm 3$ & $0.38 \pm 0.03$ & $636 \pm 5$ & $0.973 \pm 0.004$ \\
Glice 4 (G4) & 7.00 & $241 \pm 3$ & $0.43 \pm 0.03$ & $640 \pm 5$ & $0.980 \pm 0.004$ \\
Glice 5 (G5) & 9.75 & $250 \pm 3$ & $0.51 \pm 0.03$ & &
\end{tabular}

${ }^{\mathrm{a}} \mathrm{La}$ viscosidad fue medida a $200 \mathrm{rpm}$, con aguja \#34, cámara $13 \mathrm{R}$ y una temperatura de $20 \pm 3{ }^{\circ} \mathrm{C}$; ${ }^{\mathrm{b}}$ para determinar la densidad se utilizó un picnómetro de $1 \mathrm{~mL}$ y posteriormente la muestra se pesó en una balanza digital a una temperatura de $25 \pm 3{ }^{\circ} \mathrm{C}$. (Densidad del agua $1 \mathrm{~g} \mathrm{~mL}^{-1}$ ). 


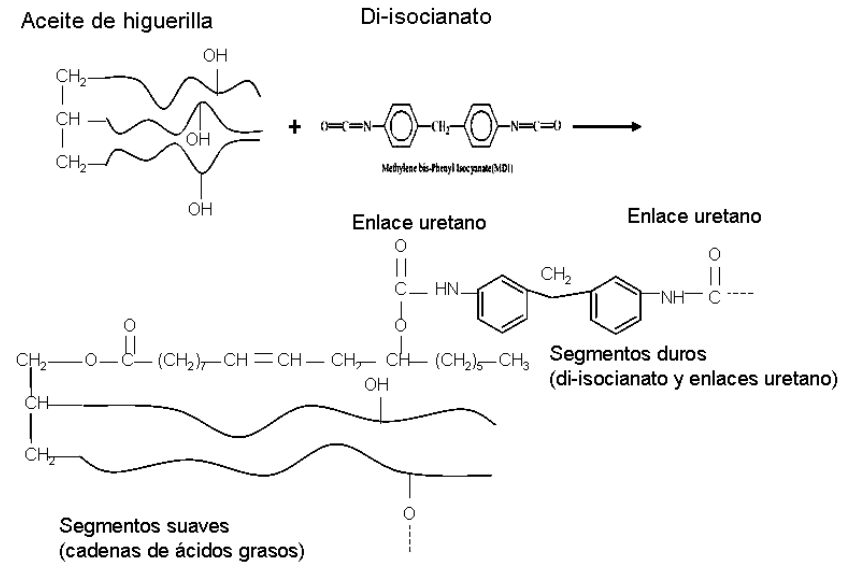

Figura 5. Esquema de obtención de la red de poliuretano a partir del aceite de higuerilla

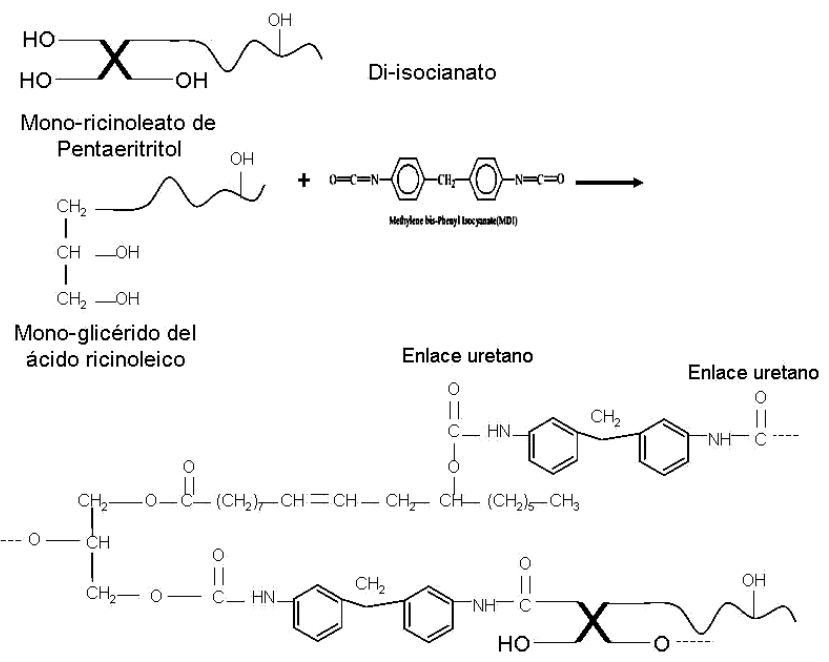

Figura 6. Esquema de obtención de la red de poliuretano a partir de los polioles obtenidos del aceite de higuerilla modificado por transesterificación

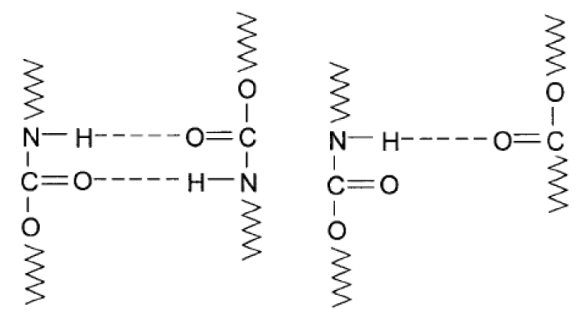

Figura 7. Posibles puentes de hidrógeno entre segmentos que contienen grupos uretano y entre segmentos que contienen grupos uretano y segmentos que contienen grupos ésteres

del poliol utilizado en la síntesis del PU disminuye la capacidad de penetración del solvente a la matriz del polímero. Al aumentar la densidad de entrecruzamiento del material, como respuesta al aumento de la funcionalidad del poliol, el material alcanza el equilibrio dinámico a un menor volumen de hinchamiento.

\section{Tensión-deformación y dureza shore A}

Los datos obtenidos de tensión de ruptura, elongación de ruptura, módulo de Young y dureza de los poliuretanos obtenidos del aceite

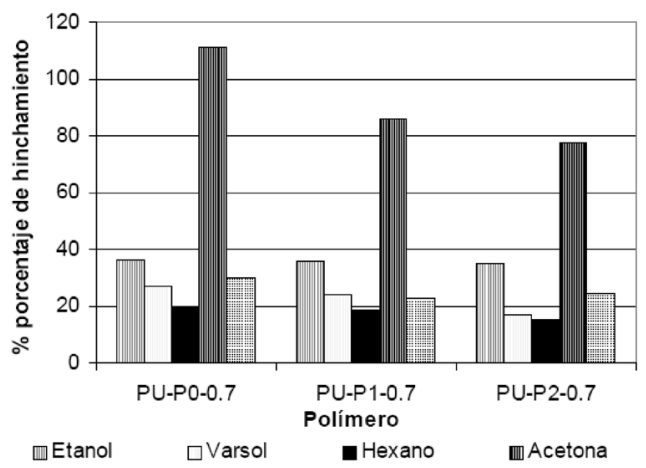

Figura 8. Porcentaje de hinchamiento de los poliuretanos sintetizados a partir del aceite modificado por transesterificación con pentaeritritol

de higuerilla sin modificar y el modificado por transesterificación con pentaeritritol y glicerol se reportan en la Tabla 4. Se observa un incremento en el módulo de elasticidad y la tensión de ruptura, a medida que se utiliza - para la síntesis del material - un poliol de mayor índice de hidroxilo. El incremento en los valores de la tensión de ruptura y módulo de Young fue: de 1960 y $226 \mathrm{KPa}$ para el poliuretano obtenido del aceite de higuerilla sin modificar (PU-CO-0.9), hasta 7323 y $1120 \mathrm{KPa}$ para el poliuretano obtenido del poliol 2(PU-P2-0.7), respectivamente. Se encontró la dureza es mayor para los poliuretanos obtenidos a partir de polioles del aceite de higuerilla modificado con pentaeritritol. Éste comportamiento se debe a que al aumentar la cantidad de grupos - $\mathrm{OH}$ del aceite de higuerilla por transesterificación aumenta la rigidez del material debido al incremento en la densidad de entrecruzamiento del poliuretano.

\section{Análisis termogravimétrico (TGA)}

Del análisis termogravimétrico se puede establecer que todos los poliuretanos sintetizados a partir del aceite de higuerilla y partir de los polioles obtenidos por transesterificación del aceite son térmicamente estables por debajo de los $200{ }^{\circ} \mathrm{C}$ (pérdida aproximada de peso entre un 2-4\%) y se descomponen completamente cerca de los $550{ }^{\circ} \mathrm{C}$. En los termogramás se presentan tres regiones de degradación. En la primera región, de 160 a $330^{\circ} \mathrm{C}$, hay una pérdida de aproximadamente el $10 \%$ en peso del material que corresponde a pérdida de humedad, hidrocarburos alifáticos lineales del aceite de higuerilla y oligómeros. En segunda región comprendida entre 340 y $450{ }^{\circ} \mathrm{C}$ existe una pérdida cercana al $40 \%$ en peso. Esta pérdida se debe a la volatilización de las ramificaciones presentes en las moléculas que se encuentran formando los enlaces uretano (secciones de ácidos, ésteres o isocianato que están unidos a la red principal,

Tabla 4. Propiedades mecánicas de los poliuretanos obtenidos a partir del aceite de higuerilla modificado por transesterificación

\begin{tabular}{lcccc}
\hline Poliuretano & $\sigma(\mathrm{KPa})^{\mathrm{a}}$ & $\in(\%)^{\mathrm{b}}$ & $\mathrm{E}(\mathrm{KPa})^{\mathrm{c}}$ & $\begin{array}{c}\text { Dureza } \\
\text { Shore A }\end{array}$ \\
\hline PU-P2-0.7 & $7323 \pm 4.9$ & $224.6 \pm 8.4$ & $1120 \pm 6.9$ & $64 \pm 2$ \\
PU-P1-0.7 & $5428 \pm 3.6$ & $219.7 \pm 7.7$ & $818 \pm 5.6$ & $60 \pm 2$ \\
PU-P0-0.7 & $4573 \pm 3.1$ & $229.5 \pm 6.8$ & $754 \pm 5.1$ & $50 \pm 2$ \\
PU-G3-0.7 & $3141 \pm 3.6$ & $249.0 \pm 6.5$ & $377 \pm 4.6$ & $50 \pm 2$ \\
PU-G2-0.7 & $1960 \pm 3.0$ & $244.1 \pm 7.3$ & $226 \pm 4.0$ & $48 \pm 2$ \\
PU-G1-0.7 & $1163 \pm 3.1$ & $214.8 \pm 6.5$ & $258 \pm 4.1$ & $38 \pm 2$ \\
PU-CO-0.9 & $553 \pm 3.1$ & $161.1 \pm 9.2$ & $201 \pm 5.1$ & $38 \pm 2$ \\
\hline
\end{tabular}

${ }^{\mathrm{a}}$ Tensión de ruptura, ${ }^{\mathrm{b}}$ Elongación de ruptura, ${ }^{\mathrm{c}}$ Módulo de Young, ${ }^{e}$ relación $\mathrm{NCO} / \mathrm{OH}=0.7$ y ${ }^{\mathrm{f}}$ relación $\mathrm{NCO} / \mathrm{OH}=0.9$ 
pero que no alcanzaron a reaccionar), así como compuestos de peso molecular promedio. Finalmente, en la tercera región se da la descomposición de las redes de poliuretano (rompimiento de enlaces uretano). Se observa también que la velocidad de degradación en la tercera región es mayor para el PU-P0-0.7 para el PU-P1-0.7. Este resultado puede ser atribuible a que al aumentar la funcionalidad del poliol se obtiene un poliuretano con una estructura de red más densa, por lo que el proceso de degradación de la red de poliuretano es más lento. Los resultados se muestran en la Figura 9(a) y Figura 9(b), respectivamente.

\section{CONCLUSIONES}

Se obtuvieron evidencias concluyentes de la transesterificación del aceite de higuerilla con pentaeritritol mediante el análisis por espectroscopia de masas MALDI TOF, en donde se muestra la obtención de un nuevo poliol. Las fracciones en masa de los productos de la reacción fue la siguiente: Aprox. 0\% tri-ricinoleína, 20.8\% di- ricinoleína, $40.5 \%$ mono-ricinoleína, $8.5 \%$ di-ricinoleato de pentaeritritol, $21.2 \%$ mono-ricinoleato de pentaeritritol y $9 \%$ componentes minoritarios. Se determinó que los polioles obtenidos por transesterificación del aceite con pentaeritritol poseen una mayor funcionalidad hidroxílica que el aceite modificado por transesterificación con glicerol y el aceite sin modificar.

Se determinó que los poliuretanos obtenidos a partir del aceite de higuerilla modificado por transesterificación con pentaeritritol poseen un mayor esfuerzo tensil y dureza Shore A que los homólogos obtenidos a partir del aceite de higuerilla modificado con glicerol y los obtenidos a partir del aceite de higuerilla original. Los ensayos de hinchamiento mostraron que al aumentar el porcentaje de pentaeritritol utilizado en la transesterificación del aceite el porcentaje de hinchamiento del poliuretano obtenido, debido a que al aumentar la funcionalidad del poliol utilizado en la síntesis aumenta la densidad de entrecruzamiento del poliuretano.

\section{AGRADECIMIENTOS}

El presente trabajo fue financiado en Colombia por SenaColciencias, a través del soporte al proyecto de investigación identificado con el código 1102-08-12731; este trabajo fue presentado por el Grupo de Investigación en Polímeros de la Universidad Industrial de Santander, en la línea de investigación sobre Desarrollo de Materiales con Aplicación Tecnológica. Los autores agradecen al Prof. Z. Cheng de la Universidad Texas A\&M por la ayuda en la realización de ensayos y pruebas de caracterización.

\section{REFERENCIAS}

1. Sperling L. H.; Mishra V.; Polymers for Advanced Technologies 1996, 7, 197.

2. Kumar, V. G.; Rama, R. M.; Guruprasad, T. R.; Rao, K. V. C.; J. Appl. Polym. Sci. 1987, 34, 1803.

3. Patel, P.; Suthar, B.; Polym. J. 1987, 23, 399.

4. Ngoc, B. T.; Vialle, J.; Quang, T. P. ; Polymer 1997, 38, 2467.

5. Somani, K.; Kansara, S.; Parmar, R.; Patel, N.; Int. J. Polym. Mater. 2004, 53, 283.

6. Sanmathi, C. S.; Prasannakumar, S.; Sherigara, B. S.; J. Appl. Polym. Sci. 2004, 94, 1029.

7. Prashantha P.; Vasanth, K.; Kumar, P.; Sherigara, B.S.; Prasannakumar, S.; Bull. Mater. Sci. 2001, 24, 535.

8. Athawale, V.; Kolekar, S.; Eur. Polym. J. 1998, 34, 1447.

9. Kaushik, A.; Paramjit, S.; Int. J. Polym.Anal. Charact. 2005, 10, 373..

10. Stübiger, G.; Pittenauer, E.; Allmaier, G.; Phytochem. Anal. 2003, 14, 337.

11. Valero, M. F.; Pulido, J. E.; Ramírez, A.; Cheng, Z.; Revista Ingeniería e Investigación 2007, 27, 51.

12. Valero, M. F.; Pulido, J. E.; Ramírez, A.; Cheng, Z.; Revista de Ingeniería Universidad de Antioquia 2007, 39, 100.

13. Valero, M. F.; Pulido, J. E.; Ramírez, A.; Cheng Z.; Revista Iberoamericana de Polímeros 2007, 8, 220. 